\title{
Regulation of proteolytic cleavage of brain- derived neurotrophic factor precursor by antidepressants in human neuroblastoma cells
}

This article was published in the following Dove Press journal:

Neuropsychiatric Disease and Treatment

I October 2015

Number of times this article has been viewed

Pao-Yen Lin ${ }^{1,2}$

'Department of Psychiatry, Kaohsiung Chang Gung Memorial Hospital, Chang Gung University College of Medicine, ${ }^{2}$ Center for Translational Research in Biomedical Sciences, Kaohsiung Chang Gung Memorial Hospital, Kaohsiung, Taiwan
Correspondence: Pao-Yen Lin Department of Psychiatry, Kaohsiung Chang Gung Memorial Hospital, 123, Dapi Road, Niaosong District, Kaohsiung City 833, Taiwan, People's Republic of China Tel +88677317123 ext 8751 Fax +88677326817 Email py1029@adm.cgmh.org.tw

\begin{abstract}
Evidence has supported the role of brain-derived neurotrophic factor (BDNF) in antidepressant effect. The precursor of BDNF (proBDNF) often exerts opposing biological effects on mature BDNF (mBDNF). Hence, the balance between proBDNF and mBDNF might be critical in total neurotrophic effects, leading to susceptibility to or recovery from depression. In the current study, we measured the protein expression levels of proBDNF, and its proteolytic products, truncated BDNF, and mBDNF, in human SH-SY5Y cells treated with different antidepressants. We found that the treatment significantly increased the production of $\mathrm{mBDNF}$, but decreased the production of truncated BDNF and proBDNF. These results support that antidepressants can promote proBDNF cleavage. Further studies are needed to clarify whether proBDNF cleavage plays a role in antidepressant mechanisms.
\end{abstract}

Keywords: antidepressant, mature BDNF, neurotrophic effect, proBDNF cleavage

\section{Introduction}

Evidence has supported the role of brain-derived neurotrophic factor (BDNF) in mediating the pathophysiology of depression. ${ }^{1}$ All $b d n f$ transcripts are translated in the endoplasmic reticulum into precursor BDNF (proBDNF) protein, which is then folded in the trans-Golgi and packaged into either the constitutive (passive) or regulated (active) secretory pathways. ${ }^{2}$ A large proportion of neuronal BDNF is secreted in the form of proBDNF, which is subsequently cleaved into mature BDNF (mBDNF) by proteases, such as plasmin or matrix metalloproteinases. ${ }^{3}$ It was found that proBDNF, working through p75 receptor, induced apoptosis in the peripheral neurons ${ }^{4}$ and facilitated long-term depression in the hippocampus. ${ }^{5}$ On the other hand, the conversion from proBDNF to mBDNF through the tissue plasminogen activator (tPA)/plasminogen cascade was shown to be essential for late-phase longterm potentiation. ${ }^{3}$ Given the opposing biological effects of proBDNF and mBDNF, including synaptic plasticity, regulation of neurogenesis, and neuronal survival, ${ }^{6,7}$ the mechanism controlling proBDNF cleavage may be an important step in regulating total neurotrophic effects in neural circuits, ${ }^{8}$ thus contributing to the pathogenesis of depression or antidepressant effect. Furthermore, these neurotrophic effects have been shown to be regulated by antidepressants. ${ }^{9}$ However, it is unclear whether antidepressant agents change the cleavage of proBDNF. In the current study, we aimed to examine the expression of different components of the proBDNF cleavage pathway under antidepressant treatment. 


\section{Methods}

\section{Cell cultures}

Human neuroblastoma SH-SY5Y cells (American Type Culture Collection (ATCC), Manassas, VA, USA) were used in all experiments. They were incubated in a humidified atmosphere of $5 \% \mathrm{CO}_{2} / 95 \%$ air at $37^{\circ} \mathrm{C}$ in Dulbecco's Modified Eagle's Medium (DMEM), 2 mM L-glutamine, $1 \%$ penicillin/streptomycin $(\mathrm{P} / \mathrm{S})$, and $10 \%(\mathrm{v} / \mathrm{v})$ fetal bovine serum. Cells were seeded at an initial density of $3 \times 10^{5}$ cells per well in the six-well culture plates in DMEM, $10 \%$ fetal bovine serum, $2 \mathrm{mM} \mathrm{L}$-glutamine, and $1 \% \mathrm{P} / \mathrm{S}$ for 24 hours, after which the medium was replaced with DMEM, 1\% N-2 supplements (Thermo Fisher Scientific, Waltham, MA, USA), $2 \mathrm{mM}$ L-glutamine, $1 \% \mathrm{P} / \mathrm{S}$, and $10 \mu \mathrm{M}$ all-trans retinoid acid (Sigma-Aldrich, St Louis, MO, USA). One-half of the medium was replaced every 2 days for up to 9 days. After the cells were plated on the culture disks for 24 hours, they were treated with three antidepressants with different pharmacodynamic properties, including bupropion (a norepinephrine-dopamine reuptake inhibitor) $1 \mu \mathrm{M}$, desipramine (a selective norepinephrine reuptake inhibitor) $1 \mu \mathrm{M}$, fluoxetine (a selective serotonin reuptake inhibitor) $1 \mu \mathrm{M}$, or without any treatment for following days until the lysates were collected. All these drugs were purchased from Sigma-Aldrich. Intracellular lysates were collected at 3 days and 9 days in vitro (DIV) for subsequent protein expression assays. The SH-SY5Y cells were undifferentiated at 3 DIV but were fully differentiated at 9 DIV. In addition, The Declaration of Helsinki was strictly followed throughout the whole process of this study.

\section{Western blot}

Western blot analysis was used for assaying protein expression. Briefly, cells were washed twice in phosphate buffered saline and then centrifuged at $2,000 \times g$ for 10 minutes. The cell pellet was lysed in a buffer containing $50 \mathrm{mM}$ Tris- $\mathrm{HCl}, \mathrm{pH}$ 7.5, $150 \mathrm{mM} \mathrm{NaCl}, 0.5 \%$ NP-40, 5 mM EDTA supplemented with a cocktail of protease inhibitors (Hoffman-La Roche Ltd., Basel, Switzerland). Cell lysates were centrifuged at $13,000 \times g$ at $4^{\circ} \mathrm{C}$ for 10 minutes. The supernatants were used for sodium dodecyl sulfate-polyacrylamide gel electrophoresis after quantification of the amount of total protein. After separation by sodium dodecyl sulfate-polyacrylamide gel electrophoresis, proteins were transferred on to a nitrocellulose membrane (Hybond; Amersham Biosciences, Amersham, UK) in transfer buffer (25 mM Tris, $192 \mathrm{mM}$ glycine, 20\% (v/v) methanol) for 2 hours at $0.4 \mathrm{~A}$ at $1{ }^{\circ} \mathrm{C}$ and blocked for 1 hour at room temperature in Tris-buffered saline-Tween-20 (TBS-T) with 5\% (w/v) nonfat milk powder (Carnation, Nestle, Vevey, Switzerland). The blot was incubated with either antibodies against BDNF $(1: 1,000)$, proBDNF $(1: 1,000)$, or beta-actin $(1: 1,000)$ (Santa Cruz Biotechnology Inc., Dallas, TX, USA) overnight at $4{ }^{\circ} \mathrm{C}$ in TBS-T with $1 \%(\mathrm{w} / \mathrm{v})$ nonfat milk powder. They were then incubated in horseradish peroxidase-conjugated donkey antigoat secondary antibodies $(1: 5,000)$ (Santa Cruz Biotechnology Inc.) in TBS-T with $1 \%$ nonfat milk powder for 1 hour at room temperature. Immunoreactive bands were detected and quantified with ImageJ software (National Institutes of Health, Bethesda, MD, USA). The proBDNF, mBDNF, truncated BDNF, and $\beta$-actin were identified at approximately $35 \mathrm{kDa}, 14 \mathrm{kDa}, 28 \mathrm{kDa}$, and $46 \mathrm{kDa}$, respectively, by $10 \%$ polyacrylamide gel.

\section{Statistical analysis}

Data for protein expression were analyzed using SPSS 15.0 (IBM, Armonk, NY, USA) software. The intensity of immunoreactive bands for BDNF protein isoforms under each drug treatment was normalized by the intensity of $\beta$-actin and was compared with the condition without drug treatment by Student's $t$-test. Difference was considered significant at $P<0.05$. Each experiment was repeated at least three times.

\section{Results and discussion}

In order to examine whether antidepressant agents regulate the proBDNF cleavage, we measured the protein expression of proBDNF, mBDNF, and truncated BDNF in human SH-SY5Y cells treated with bupropion, desipramine, or fluoxetine for 3 days or 9 days using Western blot analysis. We found that bupropion and desipramine treatments significantly increased the expression of mBDNF up to $20 \%-30 \%$ compared to the control condition. The expression of truncated BDNF was downregulated in bupropion and fluoxetine treatments for 9 days, but not in the treatment for 3 days. In addition, the expression of proBDNF was decreased in bupropion and fluoxetine treatments for 3 days, but not in the treatment for 9 days (Figure 1).

Our preliminary results showed that antidepressant agents regulate the expression of BDNF isoforms in human neuronal cells. Basically, these treatments increased the expression of mBDNF, but decreased the expression of truncated BDNF and proBDNF. Mammalian proBDNF (32 kDa) is cleaved to generate truncated BDNF (28 kDa) or mBDNF $(14 \mathrm{kDa})$ by different proteolytic enzymes. mBDNF is generated by furin intracellularly ${ }^{10}$ or by tPA/plasmin extracellularly. ${ }^{3}$ Truncated BDNF is generated by a specific calcium-dependent serine proteinase known as subtilisin/ 

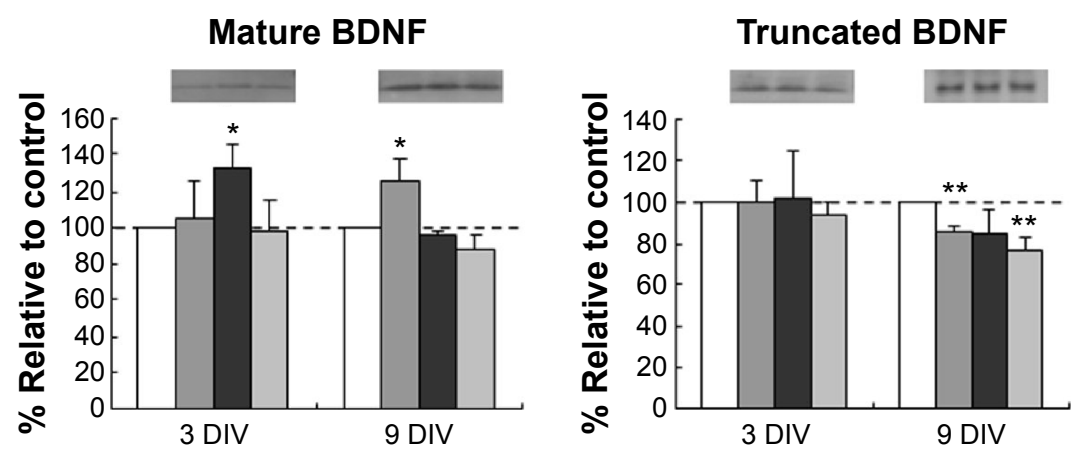

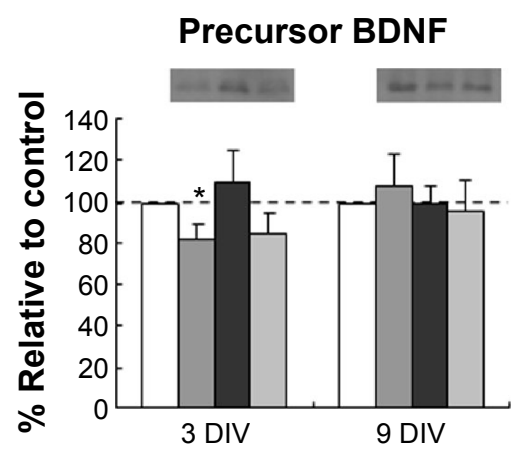

$\square$ Control $\square$ Bupropion $\square$ Desipramine $\square$ Fluoxetine

Figure I Expression of mature BDNF, truncated BDNF, and precursor BDNF in the SH-SY5Y neuronal cells treated with control, bupropion (I $\mu$ M), desipramine (I $\mu$ M), or fluoxetine (I $\mu \mathrm{M})$, for 3 days or 9 days in vitro.

Notes: The protein expression was measured by using Western blot and normalized by $\beta$-actin. The representative immunoblots were shown for respective condition. The levels of expression in cells treated with above drugs were shown as percentages relative to the control. The error bars were shown as standard deviations. $n=3-4$. $* P<0.05, * * P<0.01$

Abbreviations: BDNF, brain-derived neurotrophic factor; DIV, days in vitro.

kexin-isoenzyme. ${ }^{11}$ Our data suggest that antidepressant treatments differentially change the proBDNF cleavage, favoring the generation of $\mathrm{mBDNF}$. Increased $\mathrm{mBDNF}$ and lower proBDNF expressions in neurons enhance positive neurotrophic effects, which may be required for effective antidepressant treatment in rodent models. ${ }^{12}$

Our finding is consistent with one report showing that voluntary physical exercise robustly enhanced the expression of $\mathrm{mBDNF}$ and a proBDNF cleavage-related gene, $t P A$, in the hippocampus of mice, as well as antidepressant effects and visual-spatial learning. ${ }^{13}$ Although there was no significant change in the expression of proBDNF and truncated BDNF induced by the exercise, these findings support that the antidepressant effect of physical exercise may depend, at least in part, on the change of posttranslational proBDNF processing. ${ }^{13}$ In addition, one clinical study has examined these BDNF isoforms and their relationship with cognitive impairment in schizophrenia. ${ }^{14}$ In this study, Carlino et al found an increase in serum proBDNF and mBDNF and truncated BDNF in patients with schizophrenia. Also, measurements of serum-truncated BDNF abundance predicted high cognitive deficits. ${ }^{14}$ Although the biological effects of truncated BDNF are still unclear, these results suggest deficiency in proBDNF cleavage as one possible biochemical mechanism underlying cognitive deficits in schizophrenia.

The results in the current study need to be explained with some limitations. First, it is difficult to account for differential effects in proBDNF cleavage by bupropion, desipramine, and fluoxetine. For example, fluoxetine decreased expression of truncated BDNF only at 9 DIV, but not mBDNF and proBDNF. On the other hand, bupropion and desipramine can induce change at 3 DIV. These might be explained by the difference in the timing of expression of serotonin transporter and norepinephrine transporter in developing neurons. Second, the expression of these BDNF isoforms from extracellular medium may be valuable in delineating treatment effects on proBDNF processing, but it was not examined due to too low expression levels to be detected by Western blot.

\section{Conclusion}

In conclusion, the results of the current study support that antidepressant treatment promotes proBDNF cleavage in neuronal cells. However, it needs to be carefully confirmed in brain areas pertinent to antidepressant effects. Further studies are needed to clarify whether proBDNF cleavage plays a role in antidepressant mechanisms. If confirmed, medications that enhance the efficiency of proBDNF cleavage may provide a new treatment target for clinical depression.

\section{Acknowledgments}

This work was supported by National Science Council (NSC 99-2314-B-182A-024 -MY2) and Kaohsiung Chang Gung Memorial Hospital (CMRPG890151). They have no further role in study design, data collection, analysis and interpretation, writing of the report, or in the decision to submit the paper for publication.

\section{Author contribution}

PY Lin contributed toward data analysis, drafting and critically revising the paper and agrees to be accountable for all aspects of the work. 


\section{Disclosure}

The author reports no conflicts of interest in this work.

\section{References}

1. Duman RS, Monteggia LM. A neurotrophic model for stress-related mood disorders. Biol Psychiatry. 2006;59(12):1116-1127.

2. Mowla SJ, Pareek S, Farhadi HF, et al. Differential sorting of nerve growth factor and brain-derived neurotrophic factor in hippocampal neurons. J Neurosci. 1999;19(6):2069-2080.

3. Pang PT, Teng HK, Zaitsev E, et al. Cleavage of proBDNF by tPA/ plasmin is essential for long-term hippocampal plasticity. Science. 2004; 306(5695):487-491.

4. Teng HK, Teng KK, Lee R, et al. ProBDNF induces neuronal apoptosis via activation of a receptor complex of p75NTR and sortilin. J Neurosci. 2005;25(22):5455-5463.

5. Woo NH, Teng HK, Siao CJ, et al. Activation of p75NTR by proBDNF facilitates hippocampal long-term depression. Nat Neurosci. 2005; 8(8):1069-1077.

6. Martinowich K, Manji H, Lu B. New insights into BDNF function in depression and anxiety. Nat Neurosci. 2007;10(9):1089-1093.

7. Koshimizu H, Hazama S, Hara T, Ogura A, Kojima M. Distinct signaling pathways of precursor BDNF and mature BDNF in cultured cerebellar granule neurons. Neurosci Lett. 2010;473(3):229-232.
8. Pompili M, Serafini G, Innamorati M, et al. Agomelatine, a novel intriguing antidepressant option enhancing neuroplasticity: a critical review. World J Biol Psychiatry. 2013;14(6):412-431.

9. Pilar-Cuéllar F, Vidal R, Díaz A, et al. Neural plasticity and proliferation in the generation of antidepressant effects: hippocampal implication. Neural Plast. 2013;2013:537265.

10. Mowla SJ, Farhadi HF, Pareek S, et al. Biosynthesis and post-translational processing of the precursor to brain-derived neurotrophic factor. $J$ Biol Chem. 2001;276(16):12660-12666.

11. Seidah NG, Mowla SJ, Hamelin J, et al. Mammalian subtilisin/kexin isozyme SKI-1: a widely expressed proprotein convertase with a unique cleavage specificity and cellular localization. Proc Natl Acad Sci USA. 1999;96(4):1321-1326.

12. Shirayama Y, Chen AC, Nakagawa S, Russell DS, Duman RS. Brainderived neurotrophic factor produces antidepressant effects in behavioral models of depression. J Neurosci. 2002;22(8):3251-3261.

13. Sartori CR, Vieira AS, Ferrari EM, Langone F, Tongiorgi E, Parada CA. The antidepressive effect of the physical exercise correlates with increased levels of mature BDNF, and proBDNF proteolytic cleavagerelated genes, p11 and tPA. Neuroscience. 2011;180:9-18.

14. Carlino D, Leone E, Di Cola F, et al. Low serum truncated-BDNF isoform correlates with higher cognitive impairment in schizophrenia. J Psychiatr Res. 2011;45(2):273-279.
Neuropsychiatric Disease and Treatment

\section{Publish your work in this journal}

Neuropsychiatric Disease and Treatment is an international, peerreviewed journal of clinical therapeutics and pharmacology focusing on concise rapid reporting of clinical or pre-clinical studies on a range of neuropsychiatric and neurological disorders. This journal is indexed on PubMed Central, the 'PsycINFO' database and CAS,

\section{Dovepress}

and is the official journal of The International Neuropsychiatric Association (INA). The manuscript management system is completely online and includes a very quick and fair peer-review system, which is all easy to use. Visit http://www.dovepress.com/testimonials.php to read real quotes from published authors. 